\title{
AGROINDUSTRIALIZACIÓN DE LA ACTIVIDAD PESQUERA EN AMÉRICA LATINA
}

\author{
José Igor Hleap Zapata \\ Doctor en Ingeniería de Alimentos \\ Profesor Asociado, Universidad Nacional de Colombia \\ jihleapz@palmira.unal.edu.co
}

\section{RESUMEN}

La industria pesquera, en América Latina, nos plantea una serie de situaciones importantes relacionadas con la actividad agroindustrial tanto extractiva como productiva. Además, los procesos involucrados con la sostenibilidad y la seguridad alimentaria para los pueblos de la región son de importancia relevante y materia de análisis permanente en la búsqueda de soluciones. La disminución de los recursos, el crecimiento de la población mundial, las relaciones internacionales actuales, la globalización, el mal manejo de los alimentos debido a la poca tecnificación y conocimiento al respecto, conllevan a la necesidad de búsqueda de alternativas que permitan atenuar las dificultades alimenticias de la región. La acuicultura y el desarrollo agroindustrial para la transformación del recurso hidrobiológico son pilares fundamentales en esta búsqueda de soluciones. Es necesario plantear alternativas de elaboración de productos que se ajusten a las necesidades específicas de las regiones, que garanticen productos inocuos, de alto valor nutricional, con excelentes propiedades organolépticas y funcionales, producidos y elaborados a bajos costos y que en lo posible gocen de amplios tiempos de vida útil. Basados en esto, se plantea la fabricación de productos confeccionados con la técnica del "surimi", siendo este un producto intermedio (materia prima) representado en una pasta o pulpa de pescado neutra elaborada con la adición de crioprotectores y polifosfatos, que permite el desarrollo de múltiples alternativas alimenticias ricas en proteína tales como embutidos de diversas características tecnológicas, funcionales y de presentación, productos apanados, productos análogos o conformados, productos semipreprarados y precongelados, productos funcionales, entre otros.

\section{DESARROLLO}

En América Latina, al igual que en el ámbito internacional, la industria pesquera ha marcado, desde tiempos remotos, un eje de desarrollo económico, social, laboral, y cultural para muchos pueblos y países. Históricamente, el aporte alimenticio propiciado por los recursos hidrobiológicos ha sido importante y ha servido para el desarrollo de grandes relaciones comerciales entre las naciones.

Sin embargo, la sobreexplotación de algunas especies, la irracionalidad en el uso y explotación de las mismas, las dificultades en la infraestructura para la captura, conservación y transformación del recurso, los problemas que genera la comercialización de los peces en fresco o de los productos transformados tanto en los mercados locales como en los internacionales ha conllevado a la aparición de grandes dificultades en dicha industria manifestándose en la disminución en el consumo per cápita de dicha proteína, siendo esto más manifiesto en los países de menor desarrollo social y económico.

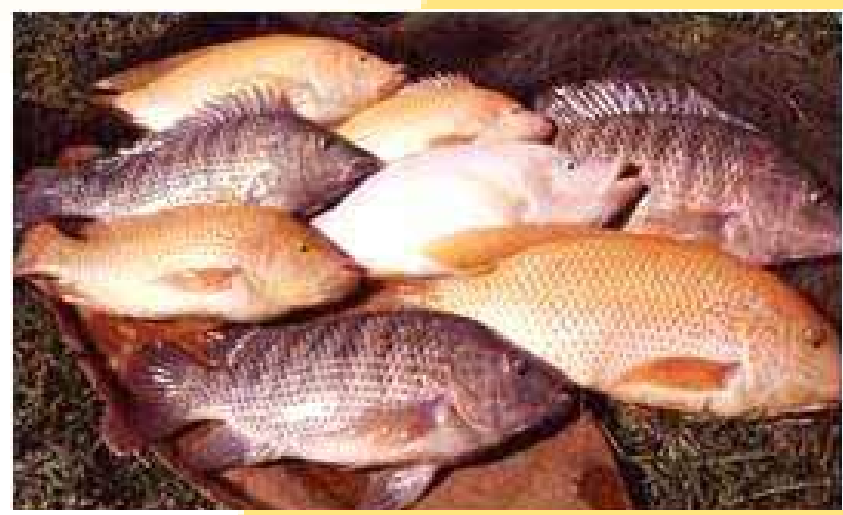

Tilapias aptas para transformación agroindustrial 


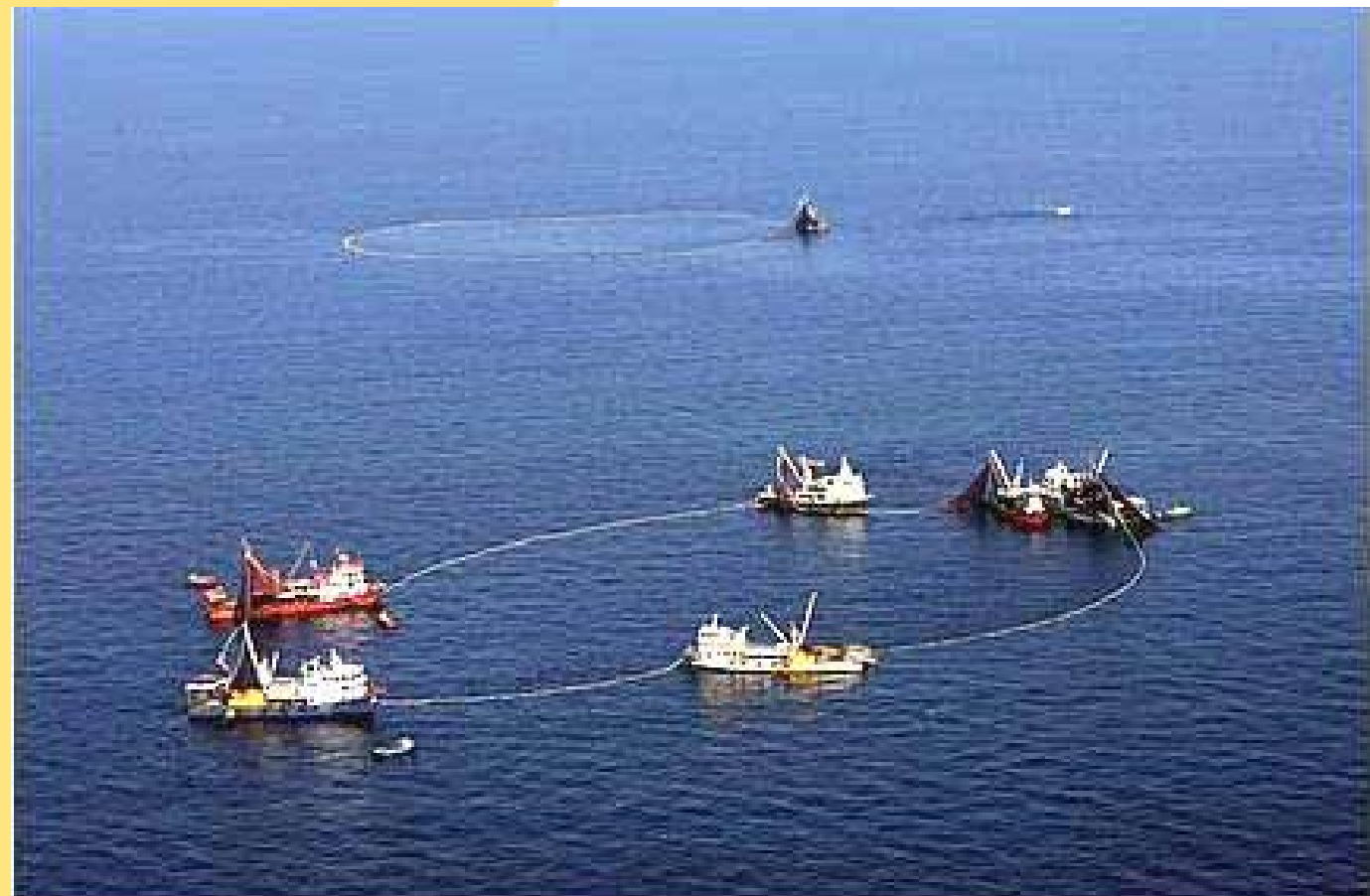

Proceso industrial de captura de peces.
La industria pesquera como una actividad integral, se puede analizar a partir de cuatro momentos importantes en su desarrollo: el conocimiento del recurso vivo, la apropiación del recurso, la conservación, elaboración $\mathrm{y}$ transformación de dicho recurso y la comercialización de los productos en fresco o transformados.
Basados en la definición propuesta por la FAO, ya hace varios años, acerca de la actividad pesquera: "Garantía de una conservación, una gestión y un desarrollo efectivo de los recursos acuáticos vivos, con el debido respeto del ecosistema y la biodiversidad, a fin de ofrecer, tanto a las generaciones presentes como venideras, una fuente de alimentos, empleo, ocio, comercio y bienestar económico", se puede apreciar como la industria pesquera extractiva puede beneficiar a la humanidad.

Sin embargo, para lograr el loable propósito de la FAO se requiere adoptar medidas importantes por parte de los dirigentes de las naciones y de las organizaciones regentes y relacionadas con la industria pesquera que conlleven a evitar desastres ecológicos tanto marinos como de aguas continentales causados en un alto porcentaje por el afán económico para el beneficio de algunos pocos, olvidándose en muchos casos de las bondades alimenticias que pueda brindar dicha industria.

Efectivamente, la actividad pesquera debe ser un polo de desarrollo para los pueblos y un polo generador de bienestar para la humanidad.
El primer aspecto es el punto de partida que nos marca las pautas para el desarrollo de una verdadera agroindustria pesquera. En efecto, el conocimiento que la humanidad ha adquirido sobre los recursos hidrobiológicos en su hábitat natural ha permitido la explotación racional de gran variedad de especies y ha sentado las bases para el desarrollo de los procesos de la acuicultura, renglón este de gran importancia hoy en día a nivel mundial.

La apropiación del recurso ha permitido industrializar la actividad pesquera al igual que el manejo de grandes volúmenes de capturas que redundan en beneficios alimenticios, sociales, económicos y de bienestar para muchas poblaciones del planeta. Igualmente, este ítem ha permitido generar desarrollos en actividades conexas tales como la navegación, las comunicaciones, la industria de frío, la teledetección de especies hidrobiológicas, la marinería, etc., lo cual ha generado desarrollo y aumento en el nivel técnico-científico del conocimiento humano.

Una vez que el recurso ha sido extraído de su medio natural, y por ser este un recurso altamente perecedero, se deben aplicar técnicas para ga- 
rantizar su conservación su elaboración previa y posteriormente su transformación a múltiples productos de diferentes cualidades nutricionales y organolépticas.

De esta misma forma se garantiza la posibilidad de que un gran número de consumidores a nivel mundial, ubicados en sitios alejados de las costas o centros de producción, tengan acceso a dichos beneficios alimenticios, elevando así su nivel de vida.

Cerrando el ciclo agroindustrial es necesario comercializar, bien sea en los mercados regionales, nacionales o internacionales, estas materias primas hidrobiológicas o bien los productos derivados, de mayor valor agregado gracias a los procesos de transformación.

Lo anterior nos muestra como la industria pesquera realmente la podemos considerar una verdadera agroindustria portadora de bienestar para el ser humano, generadora de fuentes de empleo a través de sus eslabones como son la producción, la transformación y la comercialización de los recursos hidrobiológicos.

Según datos aportados por la FAO [4] en su último boletín publicado en el año 2008, la producción pesquera continental total para el año 2006 fue de 41,7 millones de toneladas, lo cual representó un incremento, con relación al año 2001, de 24,7 \%.

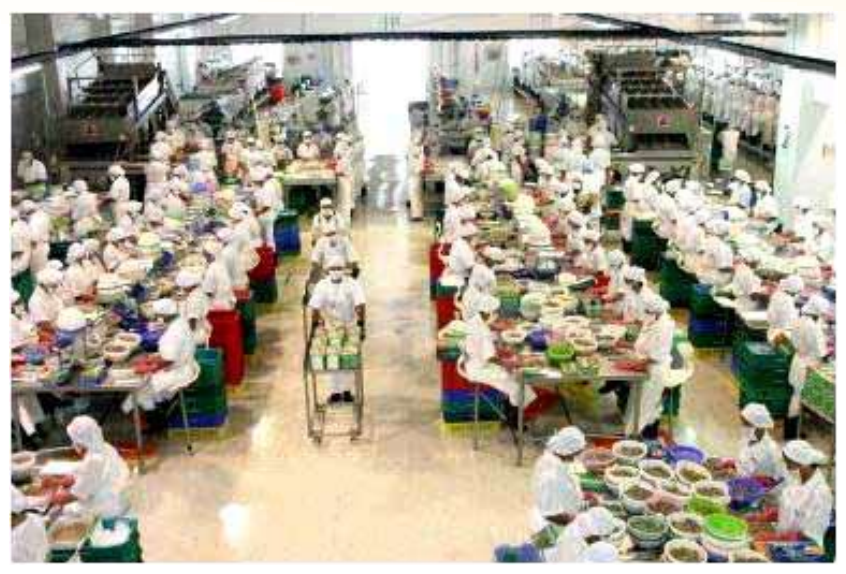

Proceso de transformación industrial del pescado
En el mismo periodo el incremento para la pesca marítima total fue de tan solo 2,35\%, alcanzado para el año 2006 un total de 102 millones de toneladas. Con relación a la acuicultura, el incremento en los mismos seis años fue de 26,7 \% llegando a ser para el año 2006 de 51,7 millones de toneladas a nivel mundial. Tomados en conjunto los tres tipos de pesquerías el incremento fue de $8,83 \%$ para los mismos seis años en mención, llegando a una producción mundial total de 143,7 millones de toneladas de pescado, siendo el continente Asiático el líder en capturas con un porcentaje total del $66,9 \%$ seguido por África con $23,5 \%$ y en menor proporción América, Europa y Oceanía.

Los datos demográficos mundiales publicados en diferentes fuentes muestran que la población a la cual la humanidad deberá alimentar para el año 2010 estará alrededor de 6.711'335.737 de habitantes, siendo China e India los mayores consumidores, debido a que sus poblaciones ya superaron los mil millones de habitantes.

Igualmente, se puede ver con cierta preocupación como la densidad de población mundial ha venido incrementándose considerablemente alcanzando para el año 2010 una proyección de 45,03 hab / $\mathrm{Km}^{2}$, lo cual comparado con 1983 (31,83 hab/ $\mathrm{Km}^{2}$ ) representa un incremento del $29,3 \%$ en 27 años [1].

Haciendo unos cálculos sencillos y a partir de los datos referidos anteriormente, se puede visualizar que la demanda de alimentos crece a una taza anual del 1,8\%. Lo anterior permite hacernos la siguiente interrogante: ¿de dónde saldrá el alimento para el futuro, si la humanidad sigue en esta tónica de crecimiento?

Varias soluciones se pueden plantear para dar respuesta a lo propuesto: mejoramiento en las tecnologías de manejo poscosecha, incremento en la utilización de las áreas no cultivadas (cada vez en menor cantidad), aumento en la producción de proteína animal con las dificultades que esto conlleva, desarrollos basados en la biotecnología 
alimentaria, mejoramiento en las tecnologías para la conservación y transformación de alimentos, entre otras.

Además de las alternativas de solución propuestas anteriormente, la humanidad cuenta con el aporte de la agroindustria pesquera, la cual ha de jugar un papel fundamental en el aporte alimenticio mundial. En efecto, este planeta está constituido en $3 / 4$ partes por agua, la cual se puede aprovechar para la producción tanto de alimento primario autótrofo, como para la producción de especies hidrobiológicas ricas en nutrientes fundamentales para el normal mantenimiento del ser humano. Nutrientes tales como proteínas completas con todos sus aminoácidos esenciales, grasas insaturadas ricas en ácidos grasos Omega 3 y Omega 6, minerales $\mathrm{y}$ algunas vitaminas.

En el ámbito Latinoamericano se dispone de una serie de recursos hidrobiológicos altamente valorados en los mercados internacionales gracias a sus bondades nutricionales, entre ellos: el bagre (Arius spp.), la cherna (Epinephelus mireatus.), la cobia (Rachycentron canadus.), el congrio (Congrina flava.), el jurel (Trachurus spp.), el lenguado (Citharichthys spilopterus.), la lisa (Mujil spp.), el pargo (Lutjanus mohogoni), el pargo rojo (Lutjanus aya.), el róbalo (Centropomus undecimales.), el sábalo (Megalus atlanticus.), la sardina (Sardinops spp.), la sierra (Scomberomorus cavalla.), los túnidos (Thunnus spp. y Katsuwonus pelamis.) y los camarones (Penaeus spp.). A esta lista es necesario agregar las especies que mayor desarrollo han obtenido a partir de los procesos de acuicultura: la tilapia (Oreochromis sp.), la cachama (Piaractus Brachypomus, Colossoma Macropomum), la trucha (Oncorhynchus Mykiss), el bocachico (Prochilodus Magdalenae), el bagre rayado (Pseudoplaatystoma Fasciatum), el salmón (Oncorhynchus sp.) entre otros.

Por otro lado, la contribución de la acuicultura al suministro mundial de pescado, crustáceos, moluscos y otros animales acuáticos ha aumentado, $\mathrm{y}$ ha pasado de un 3,9\% de la producción total en

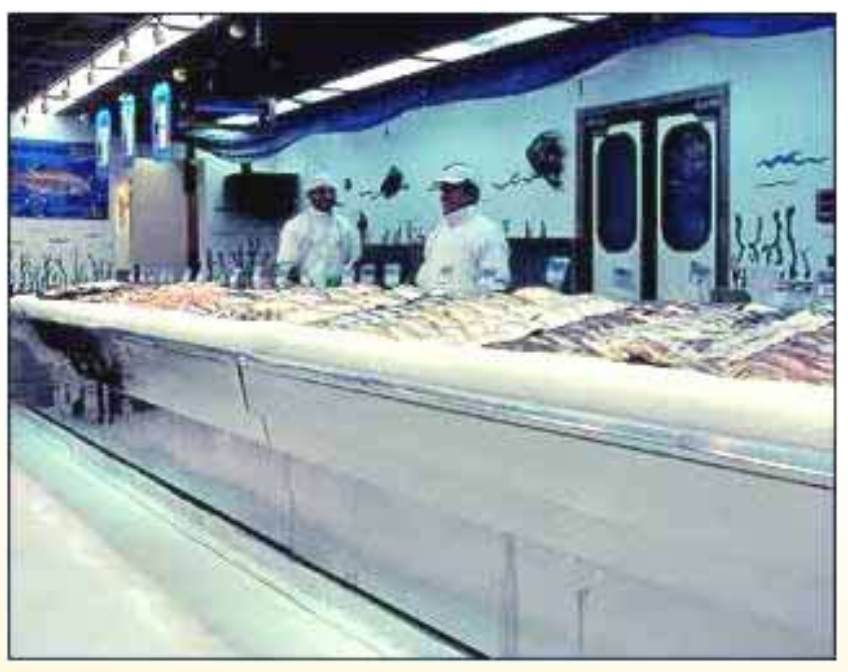

Comercialización del pescado en fresco

peso en 1970 a un $36,0 \%$ en 2006 . La acuicultura proporcionó el $47 \%$ del suministro mundial de pescado para alimentación en 2006. Mientras la producción de la pesca de captura cesó de crecer a mediados de la década de 1980, el sector acuícola ha mantenido una tasa de crecimiento medio anual del 8,7 \% en todo el mundo (excluyendo a China, con un 6,5\%) desde 1970 [4].

En un futuro cercano la contribución de las pesquerías seguirá disminuyendo y la de la acuicultura seguirá aumentando. La acuicultura es la técnica de producción de alimentos de mayor crecimiento en el mundo, y es responsable del aumento global del consumo per cápita de pescados y mariscos.

La acuicultura tiene el potencial de ayudar a aliviar la pobreza, crear trabajos, promover el desarrollo de comunidades, y disminuir la presión sobre la pesca [5].

Los datos consignados en el presente artículo, nos permiten concluir que una salida de relevancia para la agroindustria pesquera es la aplicación y desarrollo de nuevas tecnologías de procesamiento que hagan más atractivos y funcionales los productos de procedencia hidrobiológica tanto de origen extractivo como de origen productivo. 
Para el desarrollo de nuevas alternativas en productos alimenticios es necesario tener en cuenta algunas consideraciones propias del contexto mundial:

- Relación directa con las necesidades del país.

- El producto debe tener demanda entre la población

- Productos fáciles de transportar.

- Productos no caros en su producción.

- Productos que conserven el valor alimenticio durante largo tiempo.

- Productos agradables al paladar y con buenas propiedades organolépticas en general.

- Productos que respondan a las tradiciones del país o de la región.

- Conocimiento de las particularidades y características de los recursos materia prima.

- Utilización de tecnologías alcanzables en determinada región.

- Productos que permitan una utilización compleja del recurso (productos para consumo humano, productos para consumo animal, productos técnicos, médicos, químicos, veterinarios, cosmetológicos, artesanales, etc.).

- Productos que permitan la elaboración o transformación de partes del animal difíciles de comercializar en estado fresco.

Históricamente la industria pesquera se ha nutrido de productos elaborados por procedimientos tradicionales, que han venido tecnificándose gracias al crecimiento del saber humano sobre la materia.

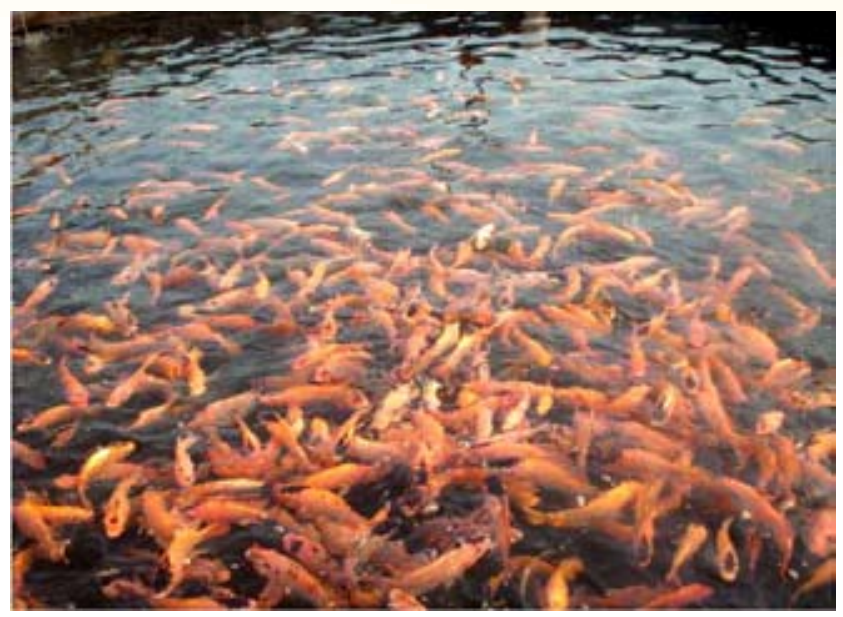

Producción de tilapia roja en estanques
Estos métodos de transformación y elaboración de productos, muchos de ellos milenarios como ya se anotó, se siguen produciendo y gozan de buena aceptación por la mayoría de los consumidores.

Entre estos podemos citar: conservación por frío, conservación por salado, conservación por deshidratación, conservación por ahumado, preservas de pescado, elaboración de conservas esterilizadas, productos de salsamentaria.

Una visión innovadora para la industria pesquera en América Latina es el desarrollo de productos para consumo directo, productos análogos o conformados y productos funcionales, elaborados a partir de una pasta base de pescado, adicionada con crioprotectores denominada Surimi (pulpa de pescado) [3].

Surimi es un término japonés que significa "músculo de pescado picado". No es un alimento en si pero sirve como materia prima para la elaboración de productos de origen hidrobiológico. El surimi es una pasta que se obtiene de pescados que por su sabor, textura, tamaño o alto contenido de grasa no se utilizan para su consumo directo [3].

En la elaboración del surimi sobresalen las siguientes características [2]:

- No tiene hueso.

- Fácil conservación, el azúcar protege al músculo de la degradación.

- Como materia prima se utiliza directamente.

- Se pueden elaborar múltiples productos a partir de esta materia prima.

Las ventajas del uso del surimi como materia prima radican en lo siguiente:

- Se reducen los procesos.

- Mejores rendimientos.

- Se asegura una calidad uniforme.

- Permite la utilización de pescados de relativo bajo valor comercial.

- Larga vida útil del producto debido a la congelación. 
- Multiplicidad y diversificación de productos elaborados.

Uno de los productos finales que se obtienen con la implementación de esta tecnología es la producción de "Kamaboko". Producto obtenido directamente del proceso de la gelatinización de la pasta de pescado (surimi) y la coagulación de ésta a base de calor.

El desarrollo de estos productos presenta una excelente alternativa para el fomento de la industria pesquera transformadora.

Se puede pensar en la elaboración de múltiples productos tales como:

- Embutidos crudos (chorizos y otros).

- Embutidos escaldados (salchichas, salchichones, mortadelas y otros).

- Embutidos cocidos (pates, jamonadas y otros). Productos no embutidos (hamburguesas, jamones prensados y otros).

- Productos semipreparados, preelaborados y congelados (apanados, croquetas, pasteles de pescado y otros).

Finalmente, se puede concluir, que la agroindustria pesquera definitivamente es un punto de desarrollo importante para el crecimiento económico y social de los países. Su aporte, en los procesos alimentarios y en la seguridad alimentaria mundial es de alta valía.

Es necesario explotar con racionalidad dichos recursos, incrementar el aporte en la materia que nos ofrece la acuicultura y trabajar en la búsqueda de nuevas especies que se puedan explotar, bien sea por la pesca extractiva o por la pesca productiva.

Paralelo a lo anterior, se debe seguir investigando en la implementación de tecnologías que permitan la creación e innovación de nuevos productos alimenticios, elaborados a bajos costos, ricos en nutrientes y en características organolépticas y funcionales.
Hacer las respectivas transferencias tecnológicas a las comunidades, propiciar el acceso de las mismas a las tecnologías de producción diseñadas bajo los cánones de calidad establecidos por los organismos de control internacional, favorecer la comercialización de los productos tanto en los mercados nacionales como en los internacionales y garantizar la sustentabilidad de la industria pesquera para las generaciones venideras.

\section{BIBLIOGRAFÍA}

- García F., J.C. Demografía mundial: tendencias recientes. 2005. Universidad Complutense de Madrid, Madrid, 2005. Documento virtual disponible en: http://www.vinculando. org/economía_solidaria/demografía_mundial. html.

- Hleap Z., J.I. Guía Práctica de laboratorio. Curso: Agroindustria de Productos Pesqueros. Universidad Nacional de Colombia - sede Palmira. Palmira, Valle del Cauca, 2007.

- Hleap Z., J.I., Molina C., A. Proceso de elaboración de salchichas a partir de tilapia roja (Oreochromis sp.) con adición de almidón de sagú (Marantha arundinacea). Manual de Transferencia Tecnológica. Universidad Nacional de Colombia - sede Palmira. ISBN 978-958-8095-52-3. Palmira, Valle del Cauca, 2009.

- Organización de las Naciones Unidas para la Agricultura y la Alimentación. El estado mundial de la pesca y la acuicultura 2008. FAO - Departamento de Pesca y Acuicultura de la Fao, Roma, 2009. Documento virtual disponible en http://www.fao.org.

- Torres Q., E. Estado actual de la acuicultura. Documento virtual disponible en: http://www. acuiculturaaldia.com/Documnetos/Estado $\%$ 20 actual $\% 20$ Acuicultura $\% 20 \mathrm{y} \% 20$ Mercado. pdf. 